\title{
Application Article \\ Using Truncated Data Sets in Spherical-Scanning Antenna Measurements
}

\author{
Ronald C. Wittmann, Carl F. Stubenrauch, and Michael H. Francis
}

Electromagnetics Division, National Institute of Standards and Technology, 325 Broadway, Boulder, CO 80305, USA

Correspondence should be addressed to Ronald C. Wittmann, wittmannrc@gmail.com

Received 12 October 2011; Accepted 8 February 2012

Academic Editor: Amedeo Capozzoli

Copyright ( $) 2012$ Ronald C. Wittmann et al. This is an open access article distributed under the Creative Commons Attribution License, which permits unrestricted use, distribution, and reproduction in any medium, provided the original work is properly cited.

We discuss the mitigation of truncation errors in spherical-scanning measurements by use of a constrained least-squares estimation method. The main emphasis is the spherical harmonic representation of probe transmitting and receiving functions; however, our method is applicable to near-field measurement of electrically small antennas for which full-sphere data are either unreliable or unavailable.

\section{Introduction}

The transmitting function of an electrically small probe tends to be very broad, so that full-sphere data are needed to compute the spherical-harmonic representation of the pattern by use of standard methods [1]. Unfortunately, backward-hemisphere data are often unavailable or at best unreliable due to support-structure blockage. Simply setting the backward-hemisphere data to zero leads to ringing effects that degrade the accuracy of the probe-pattern representation in the forward hemisphere. In this paper, we approximate the spherical-harmonic expansion of transmit/receive functions using only forward hemisphere data. The solution presented is a least-squares fit with energy constraints (to limit backward-hemisphere radiation) and proper weighting of measurements (to compensate for clustering near the poles). The energy constraint is specified using an estimated value of directivity. Results are given that compare pattern representations obtained using both full-sphere and halfsphere data.

Others have also considered truncation error mitigation in various near-field measurement contexts [2-5].

\section{Theory}

We wish to approximate a physical quantity $\mathbf{b}$ by a sphericalharmonic expansion

$$
\begin{aligned}
\mathbf{b}(\theta, \varphi) & =b^{\theta}(\theta, \varphi) \hat{\boldsymbol{\theta}}+b^{\varphi}(\theta, \varphi) \hat{\boldsymbol{\varphi}} \approx \hat{\mathbf{b}}(\theta, \varphi), \\
\hat{\mathbf{b}}(\theta, \varphi) & =\sum_{\mu=-M}^{M} \sum_{\nu=n(\mu)}^{N}\left[a_{\nu \mu}^{1} \mathbf{X}_{\nu \mu}(\theta, \varphi)+a_{\nu \mu}^{2} \mathbf{Y}_{\nu \mu}(\theta, \varphi)\right], \\
n(\mu) & =\max (1,|\mu|), \quad|M| \leq N .
\end{aligned}
$$

Here, $\mathbf{X}_{n m}$ and $\mathbf{Y}_{n m}=\hat{i} \times \mathbf{X}_{n m}$ are vector spherical harmonics [6, chapter 16]. For example, $\mathbf{b}(\theta, \varphi)$ might be the measured transmitting or receiving pattern of a probe, or it might be the "measurement vector" $\mathbf{w}(\theta, \varphi)$ for a spherical near-field scanning measurement [1,7-9].

When full-sphere data are available, the orthogonality of vector spherical harmonics can be used to determine the coefficients $a_{\nu \mu}^{1}$ and $a_{\nu \mu}^{2}$ from discrete measurements of $\mathbf{b}(\theta, \varphi)$ gathered on a uniform grid in $\theta$ and $\varphi$. When so determined, $\widehat{\mathbf{b}}(\theta, \varphi)$ is optimal in the sense that

$$
\int_{0}^{\pi} \sin \theta d \theta \int_{0}^{2 \pi}|\mathbf{b}(\theta, \varphi)-\hat{\mathbf{b}}(\theta, \varphi)|^{2} d \varphi
$$

is minimal. 
In this paper, we assume that data are available only in the forward hemisphere $\theta \leq \pi / 2$ on the grid

$$
\begin{aligned}
& \theta_{n}=\frac{\pi}{2 P^{\prime}} n, \quad 0 \leq n \leq P^{\prime}, \\
& \varphi_{m}=\frac{2 \pi}{Q} m, \quad 0 \leq n<Q,
\end{aligned}
$$

with

$$
\begin{gathered}
P^{\prime}>N, \\
Q>2 M .
\end{gathered}
$$

Requirement (5) preserves the standard number of measurement points by halving the maximum sample interval in $\theta$. Ideally, $P^{\prime}$ and $Q$ are products of small primes to allow efficient application of the fast Fourier transform (FFT) algorithm; however, factorization may not be important when antennas are "electrically small." Our goal is to minimize the discrepancy

$$
\Delta=\sum_{n=0}^{P^{\prime}} w_{n} \int_{0}^{2 \pi}\left|\mathbf{b}\left(\theta_{n}, \varphi\right)-\widehat{\mathbf{b}}\left(\theta_{n}, \varphi\right)\right|^{2} d \varphi,
$$

where the $w_{n}$ are positive weights. Equation (6) may be viewed as an adaptation of (3). Because the sampling theorem has been satisfied, the $\varphi$ integral can be readily evaluated analytically. The $\theta$ integral, however, has been discretized. (We generally set $w_{n}=\sin \theta_{n}$ ).

The discrepancy $\Delta$ is minimized subject to the "energy" constraint

$$
E=\sum_{\nu \mu}\left(\left|a_{\nu \mu}^{1}\right|^{2}+\left|a_{\nu \mu}^{2}\right|^{2}\right)
$$

in order to limit the power radiated into directions where there are no measurements. When $\mathbf{b}(\hat{\mathbf{r}})$ is proportional to the transmitting pattern of the test antenna, we have

$$
E=\frac{4 \pi|\mathbf{b}(\hat{\mathbf{r}})|^{2}}{D(\widehat{\mathbf{r}})}
$$

so that $E$ may be chosen using an estimate of the directivity $D$ in some measurement direction $\widehat{\mathbf{r}}$. (Directivity and gain are approximately equal when ohmic losses are small).

Avoiding details for the moment, we write

$$
\mathbf{b} \approx \widehat{\mathbf{b}}=\mathbf{A x},
$$

where $\mathbf{b}$ and $\hat{\mathbf{b}}$ are vectors of measurements and predicted values, $\mathbf{x}$ is a vector of coefficients to be determined, and the matrix A represents a known linear relationship. We choose $\mathbf{x}$ to minimize $\Delta=(\mathbf{b}-\mathbf{A x})^{*} \boldsymbol{\kappa}(\mathbf{b}-\mathbf{A x})$ subject to the constraint $E=\|\mathbf{x}\|^{2}$. Here, $\boldsymbol{\kappa}$ is a diagonal matrix of positive weights, and an asterisk implies Hermitian transpose. It is easy to show that this $\mathbf{x}$ is determined by the equations

$$
\begin{aligned}
\mathbf{A}^{*} \boldsymbol{\kappa} \mathbf{A} \mathbf{x}-\lambda \mathbf{x} & =\mathbf{A}^{*} \boldsymbol{\kappa} \mathbf{b}, \\
\mathbf{x}^{*} \mathbf{x} & =E
\end{aligned}
$$

The significance of the Lagrange multiplier $\lambda$ is seen in the relation

$$
|\lambda|=\frac{\left\|\mathbf{A}^{*} \boldsymbol{\kappa} \mathbf{A} \mathbf{x}-\mathbf{A}^{*} \boldsymbol{\kappa} \mathbf{b}\right\|}{\sqrt{E}}
$$

that is, $|\lambda|$ is a measure of the residual of the fit. In the absence of the energy constraint (7) of course, we can choose $\mathbf{x}$ to be the solution of the normal equations $\mathbf{A}^{*} \boldsymbol{\kappa} \mathbf{A x}=\mathbf{A}^{*} \boldsymbol{\kappa} \mathbf{b}$.

Since $\mathbf{A}^{*} \boldsymbol{\kappa} \mathbf{A}$ is Hermitian nonnegative definite, we have the singular-value decomposition (SVD)

$$
\mathbf{A}^{*} \boldsymbol{\kappa} \mathbf{A}=\mathbf{S D S}^{*} \text {, }
$$

where $S$ is unitary, $D_{i j}=d_{i} \delta_{i j}$, and $d_{i} \geq 0$. From (13), (10), and (11), we are led to

$$
\begin{aligned}
E & =\sum_{i} \frac{\left|f_{i}\right|^{2}}{\left(d_{i}-\lambda\right)^{2}}, \\
\mathbf{f} & \equiv \mathbf{S}^{*} \mathbf{A}^{*} \boldsymbol{\kappa} \mathbf{b} .
\end{aligned}
$$

The right side of (14) tends to 0 as $|\lambda| \rightarrow \infty$, and because of poles at the singular values $d_{i}$, there may be numerous solutions for $\lambda$. According to (12), the best choice corresponds to the unique solution for which $\lambda<d_{1}$, where $d_{1}$ is the smallest singular value. In general, $\lambda<0$, since the constrained solution is expected to have lower energy than the unconstrained solution. Finally, given $\lambda$, we may compute $\mathbf{x}$,

$$
x_{i}=\sum_{j} \frac{S_{i j} f_{j}}{d_{j}-\lambda} .
$$

Numerical methods used in this paper are discussed in greater detail in [10].

At this point, we have discussed the main ideas of this paper. What follows are some rather unpleasant details that we summarize dutifully and concisely. To begin with, (1) can be written as a Fourier series

$$
\mathbf{b}(\theta, \varphi)=\sum_{\mu=-M}^{M}\left[b_{\mu}^{\theta}(\theta) \hat{\boldsymbol{\theta}}+b_{\mu}^{\varphi}(\theta) \hat{\boldsymbol{\varphi}}\right] \exp (i \mu \varphi),
$$

where the coefficients may be computed from the data with a discrete Fourier transform

$$
b_{\mu}^{\theta, \varphi}(\theta)=\frac{1}{Q} \sum_{m=0}^{Q-1} b^{\theta, \varphi}\left(\theta, \varphi_{m}\right) \exp \left(-i \frac{2 \pi}{Q} m \mu\right) .
$$

Similarly, for (2),

$$
\begin{aligned}
\hat{\mathbf{b}}(\theta, \varphi) & =\sum_{\mu=-M}^{M}\left[\hat{b}_{\mu}^{\theta}(\theta) \hat{\boldsymbol{\theta}}+\hat{b}_{\mu}^{\varphi}(\theta) \hat{\boldsymbol{\varphi}}\right] \exp (i \mu \varphi), \\
\hat{b}_{\mu}^{\theta, \varphi}(\theta) & =\sum_{\nu=n(\mu)}^{N}\left[a_{\nu \mu}^{1} X_{\nu \mu}^{\theta, \varphi}(\theta, 0)+a_{\nu \mu}^{2} Y_{\nu \mu}^{\theta, \varphi}(\theta, 0)\right], \\
\mathbf{X}_{\nu \mu} & =X_{\nu \mu}^{\theta} \hat{\boldsymbol{\theta}}+X_{\nu \mu}^{\varphi} \hat{\boldsymbol{\varphi}}, \\
\mathbf{Y}_{\nu \mu} & =Y_{\nu \mu}^{\theta} \hat{\boldsymbol{\theta}}+Y_{\nu \mu}^{\varphi} \hat{\boldsymbol{\varphi}}, \\
Y_{\nu \mu}^{\theta} & =-i X_{\nu \mu}^{\varphi}, \quad Y_{\nu \mu}^{\varphi}=i X_{\nu \mu}^{\theta} .
\end{aligned}
$$


With substitution of (16) and (18), the discrepancy (6) becomes

$\Delta=2 \pi \sum_{n=0}^{P^{\prime}} w_{n} \sum_{\mu=-M}^{M}\left[\left|b_{\mu}^{\theta}\left(\theta_{n}\right)-\hat{b}_{\mu}^{\theta}\left(\theta_{n}\right)\right|^{2}+\left|b_{\mu}^{\varphi}\left(\theta_{n}\right)-\hat{b}_{\mu}^{\varphi}\left(\theta_{n}\right)\right|^{2}\right]$.

This may be rewritten in the form

$$
\Delta=2 \pi \sum_{\mu=-M}^{M}\left(\mathbf{b}_{\mu}-\mathbf{A}_{\mu} \mathbf{x}_{\mu}\right)^{*} \boldsymbol{\kappa}_{\mu}\left(\mathbf{b}_{\mu}-\mathbf{A}_{\mu} \mathbf{x}_{\mu}\right) .
$$

Here

$$
\begin{aligned}
& \mathbf{x}_{\mu}=\left(\begin{array}{c}
\mathbf{x}_{\mu}^{1} \\
\mathbf{x}_{\mu}^{2}
\end{array}\right), \quad \mathbf{b}_{\mu}=\left(\begin{array}{c}
\mathbf{b}_{\mu}^{\theta} \\
\mathbf{b}_{\mu}^{\varphi}
\end{array}\right), \\
& \mathbf{x}_{\mu}^{1,2}=\left(\begin{array}{c}
a_{n(\mu), \mu}^{1,2} \\
\vdots \\
a_{N \mu}^{1,2}
\end{array}\right), \quad \mathbf{b}_{\mu}^{\theta, \varphi}=\left(\begin{array}{c}
b_{\mu}^{\theta, \varphi}\left(\theta_{0}\right) \\
\vdots \\
b_{\mu}^{\theta, \varphi}\left(\theta_{P^{\prime}}\right)
\end{array}\right), \\
& \mathbf{A}_{\mu}=\left(\begin{array}{cc}
\boldsymbol{\alpha}_{\mu} & \boldsymbol{\beta}_{\mu} \\
i \boldsymbol{\beta}_{\mu} & i \boldsymbol{\alpha}_{\mu}
\end{array}\right), \\
& \boldsymbol{\alpha}_{\mu}=\left(\begin{array}{ccc}
X_{n(\mu), \mu}^{\theta}\left(\theta_{0}, 0\right) & \cdots & X_{N \mu}^{\theta}\left(\theta_{0}, 0\right) \\
\vdots & \ddots & \vdots \\
X_{n(\mu), \mu}^{\theta}\left(\theta_{P^{\prime}}, 0\right) & \cdots & X_{N \mu}^{\theta}\left(\theta_{P^{\prime}}, 0\right)
\end{array}\right), \\
& \boldsymbol{\beta}_{\mu}=-i\left(\begin{array}{ccc}
X_{n(\mu), \mu}^{\varphi}\left(\theta_{0}, 0\right) & \cdots & X_{N \mu}^{\varphi}\left(\theta_{0}, 0\right) \\
\vdots & \ddots & \vdots \\
X_{n(\mu), \mu}^{\varphi}\left(\theta_{P^{\prime}}, 0\right) & \cdots & X_{N \mu}^{\varphi}\left(\theta_{P^{\prime}}, 0\right)
\end{array}\right) \text {, } \\
& \boldsymbol{\kappa}_{\mu}=\left(\begin{array}{ll}
\mathbf{w} & \mathbf{0} \\
\mathbf{0} & \mathbf{w}
\end{array}\right), \\
& \mathbf{w}=\left(\begin{array}{cccc}
w_{0} & 0 & \cdots & 0 \\
0 & w_{1} & \ddots & \vdots \\
\vdots & \ddots & \ddots & 0 \\
0 & \cdots & 0 & w_{P^{\prime}}
\end{array}\right) .
\end{aligned}
$$

Finally, with the definitions

$$
\mathbf{A}=\left(\begin{array}{cccc}
\mathbf{A}_{-M} & \mathbf{0} & \cdots & \mathbf{0} \\
\mathbf{0} & \mathbf{A}_{-M+1} & \ddots & \vdots \\
\vdots & \ddots & \ddots & \mathbf{0} \\
\mathbf{0} & \cdots & \mathbf{0} & \mathbf{A}_{M}
\end{array}\right),
$$

$$
\boldsymbol{\kappa}=\left(\begin{array}{cccc}
\boldsymbol{\kappa}_{-M} & \mathbf{0} & \cdots & \mathbf{0} \\
\mathbf{0} & \boldsymbol{\kappa}_{-M+1} & \ddots & \vdots \\
\vdots & \ddots & \ddots & \mathbf{0} \\
\mathbf{0} & \cdots & \mathbf{0} & \boldsymbol{\kappa}_{M}
\end{array}\right)
$$

$$
\mathbf{x}=\left(\begin{array}{c}
\mathbf{x}_{-M} \\
\vdots \\
\mathbf{x}_{M}
\end{array}\right), \quad \mathbf{b}=\left(\begin{array}{c}
\mathbf{b}_{-M} \\
\vdots \\
\mathbf{b}_{M}
\end{array}\right) \text {, }
$$

we have

$$
\begin{gathered}
\mathbf{b} \approx \hat{\mathbf{b}}=\mathbf{A} \mathbf{x}, \\
E=\|\mathbf{x}\|^{2}, \\
\Delta=(\mathbf{A} \mathbf{x}-\mathbf{b})^{*} \boldsymbol{\kappa}(\mathbf{A} \mathbf{x}-\mathbf{b}) .
\end{gathered}
$$

Thus, the measurement truncation problem has been reduced, explicitly, to the constrained least-square optimization problem discussed earlier. In particular, (10) and (11) can be written as

$$
\begin{gathered}
\mathbf{A}_{\mu}^{*} \boldsymbol{\kappa}_{\mu} \mathbf{A}_{\mu} \mathbf{x}_{\mu}-\lambda \mathbf{x}_{\mu}=\mathbf{A}_{\mu}^{*} \boldsymbol{\kappa}_{\mu} \mathbf{b}_{\mu}, \quad-M \leq \mu \leq M, \\
E=\sum_{\mu=-M}^{M}\left\|\mathbf{x}_{\mu}\right\|^{2} .
\end{gathered}
$$

Because of the block-diagonal structure, the overall SVD can be broken into smaller parts, one $\mu$ at a time, resulting in an algorithm with computational complexity $\mathcal{O}\left(N^{4}\right)$. Comparing unfavorably with the $\mathcal{O}\left(\mathrm{N}^{3}\right)$ complexity for the full-sphere case, our method may be less useful for larger antennas. The order of $\mathbf{A}_{\mu}$ is $2\left(P^{\prime}+1\right) \times 2(N-n(\mu)+1)$, so there will be more equations than unknowns in every block $|\mu| \leq M$ as long as $P^{\prime} \geq N$. Actually, there is always a unique solution to our constrained optimization problem; however, the quality of this solution is observed to degrade dramatically as $P^{\prime}$ is decreased below the threshold $P^{\prime}=N$.

\section{Experimental and Simulated Results}

To investigate the utility of our least-squares technique for reducing the truncation error that results from zero filling in the rear hemisphere, we examined a number of typical probes for which full-sphere far-field patterns are available. In each case, we computed the far-field pattern using the spherical mode expansion obtained (a) from the standard algorithm with full-sphere data, (b) from the standard algorithm with zero-fill in the backward-hemisphere, and finally, (c) from the constrained least-squares fit to forwardhemisphere measurements described previously. Ideally, the far-field pattern calculated from the spherical-mode expansion should agree with the original pattern. For the fullsphere modal calculation, this is of course the case if the sampling theorem is satisfied. We show E-plane results only, 
since truncation effects are more important because the patterns tend to be broader. For all cases, the sampling is $2 \circ$ in $\theta$ and $5 \circ$ in $\phi$.

3.1. High-Gain Symmetric Probe. In this case, the input data were simulated using a far-field pattern calculated from a specified set of $(m= \pm 1)$ modal coefficients. For this antenna, we used $N=10, M=5$. The directivity of $15.44 \mathrm{~dB}$ was used in (8) to determine the energy constraint. Figures 1 and 2 indicate excellent agreement between the full-sphere and least-squares techniques in the forward hemisphere.

In this example, the constrained least-squares technique also performs well in the backward-hemisphere if the correct energy is specified. This is not necessarily true in the presence of noise. As a test, we added random errors with an RMS value of $0.1 \%$ relative to the noise-free RMS pattern level. An unconstrained fit resulted in an on-axis directivity of $-32 \mathrm{~dB}$. In other words, the back hemisphere overwhelmed the forward hemisphere. On the other hand, a constrained fit forced the back hemisphere pattern to remain at a reasonable level even though the details were incorrect. Although an unconstrained fit may be somewhat better in the forward directions, a constrained fit is undoubtedly more reasonable given our knowledge of the directivity of the test antenna.

In probe-corrected spherical near-field measurements, for example, the complete probe pattern is required. We often argue that the forward hemisphere pattern is most important, but all bets are off when the back hemisphere pattern dwarfs the forward hemisphere pattern.

3.2. NIST Circularly Cylindrical Waveguide Probe. This cylindrical waveguide probe was designed and built for spherical near-field measurements at $3.3 \mathrm{GHz}$. The far-field pattern was measured over the entire sphere, although supportstructure blockage limits the value of backward-hemisphere information. In this case, we used $N=17, M=17$. A directivity of $7.87 \mathrm{~dB}$ was calculated using the full-sphere data. Deviations (Figure 3) between the full-sphere and zerofill methods will lead to significant errors when the zerofill results are used in spherical scanning measurements. Within the range $\pm 75^{\circ}$, the maximum error in the zerofill result is about $0.6 \mathrm{~dB}$, while the least-squares technique has a maximum error of $0.08 \mathrm{~dB}$. Figure 4 shows how the full-sphere, zero-fill, and least-squares results compare in the backward-hemisphere. In this case, the truncation level is as high as $-12 \mathrm{~dB}$, which causes a greater discrepancy between the zero-fill and full-sphere patterns than observed in the previous example.

3.3. Rectangular Waveguide Probe. This probe is a section of WR-284 rectangular waveguide. Far-field patterns were obtained over the entire sphere at $3.3 \mathrm{GHz}$. In this case, we used $N=17, M=17$. Strictly speaking, rectangular waveguide probes do not have the correct symmetry for spherical near-field scanning applications; however, they often perform satisfactorily when the scan radius is more than about an antenna diameter. Thus, we include results for this type of probe. A directivity of $7.12 \mathrm{~dB}$, calculated from the full-sphere modal expansion, was used to determine the

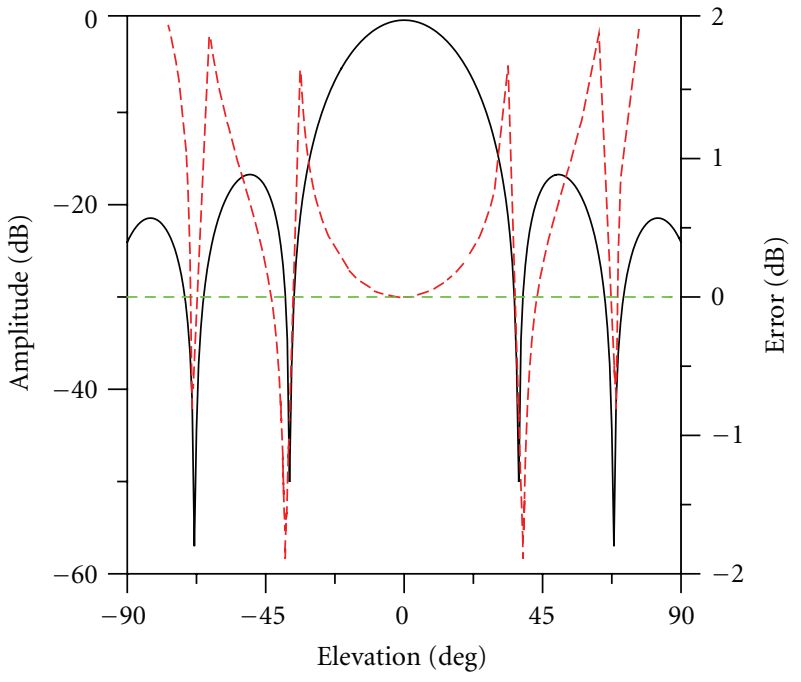

FIGURE 1: Simulated probe: deviations from the full-sphere pattern (errors) are shown for the zero-fill (long dash) and least-squares (short dash) techniques. The full-sphere pattern is shown as a solid line.

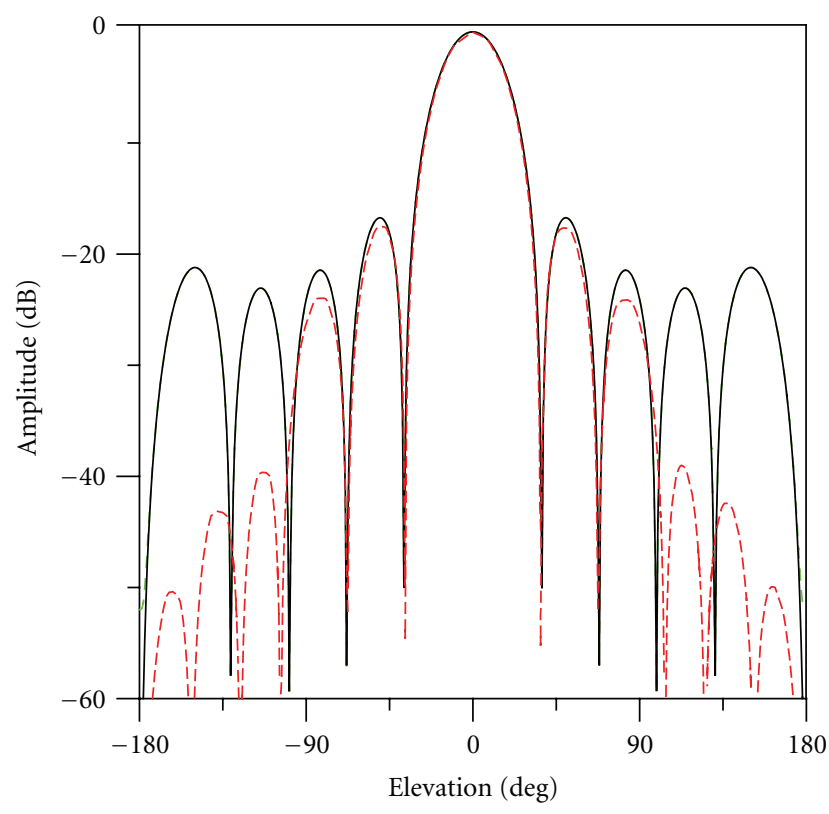

FIGURE 2: Simulated probe: comparison of the full-sphere Eplane far-field patterns obtained using zero-fill (long dash) and least-squares (short dash) techniques. The full-sphere pattern is shown as a solid line. The least-squares and full-sphere patterns are indistinguishable at this scale.

energy constraint. Measured gain was $6.7 \mathrm{~dB}$. The results are similar to those for the cylindrical waveguide case. As evident in Figure 5, within the angular range $\pm 75^{\circ}$, the zero-fill error is greatest at about $40^{\circ}$, where it is $0.66 \mathrm{~dB}$. The error is only about $0.11 \mathrm{~dB}$ for the least-squares technique at this angle. In the forward hemisphere, the zero-fill technique exhibits large errors, while the least-squares technique errors remain 


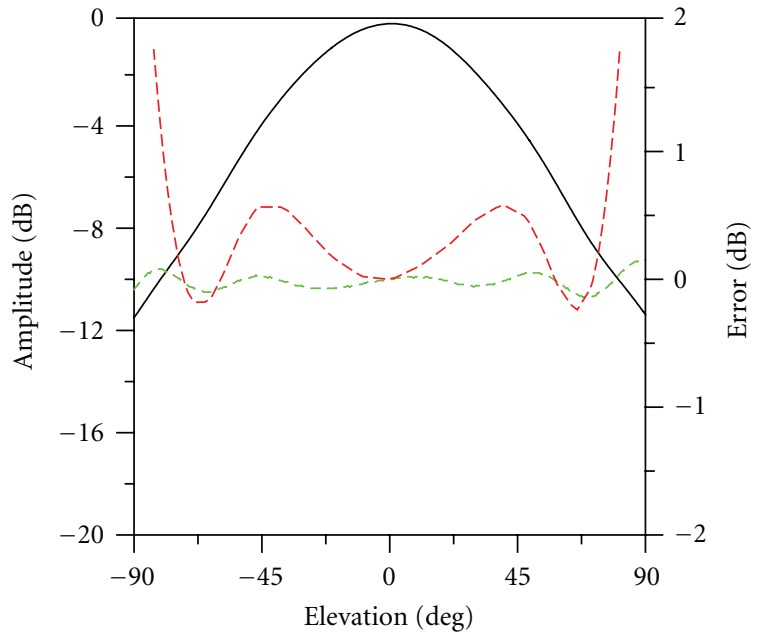

Figure 3: Cylindrical waveguide probe: deviations from the fullsphere pattern (errors) are shown for the zero-fill (long dash) and least-squares (short dash) techniques. The full-sphere pattern is shown as a solid line.

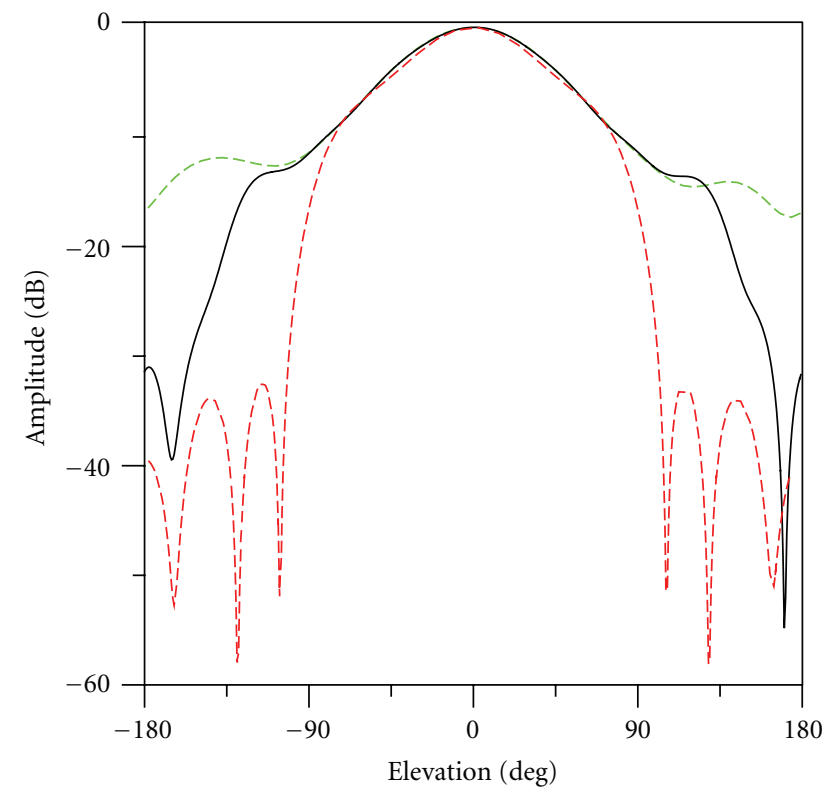

FIGURE 4: Cylindrical waveguide probe: comparison of the fullsphere E-plane far-field patterns obtained using the zero-fill (long dash) and the least-squares (short dash) techniques. The full-sphere pattern is shown as a solid line.

relatively small. Figure 6 provides a full-sphere comparison of the three techniques.

3.4. Choice of E. Several tests indicate that the quality of the least-squares fit in the forward hemisphere is relatively insensitive to the choice of $E$. In a typical case with $0.9 E_{0} \leq$ $E \leq 1.1 E_{0}$, the errors observed in the results were generally much less than $0.15 \mathrm{~dB}$ within $20^{\circ}$ of the on-axis direction. (Here, $E_{0}$ is the correct value). When gain is used to

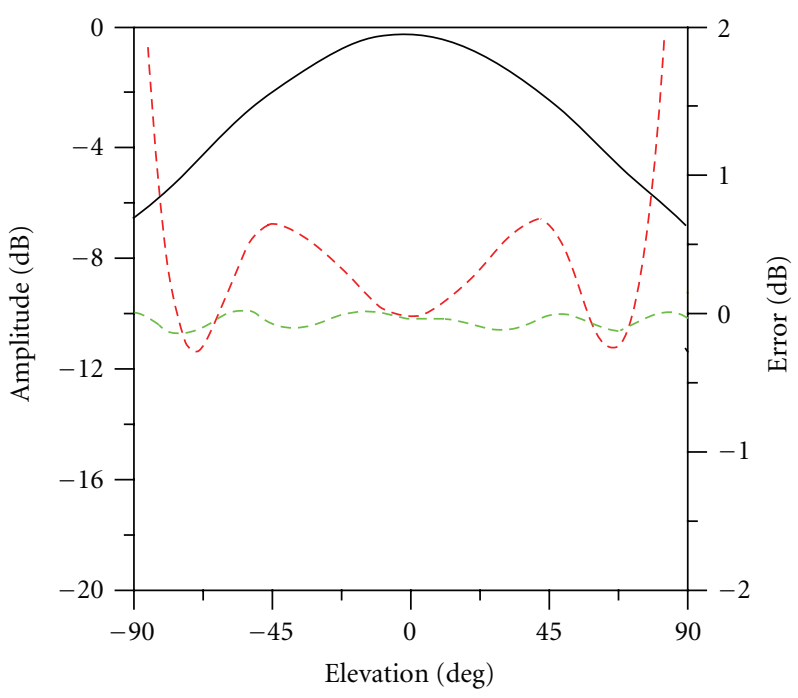

FIGURE 5: Rectangular waveguide probe: deviations from the fullsphere pattern (errors) are shown for the zero-fill (long dash) and least-squares (short dash) techniques. The full-sphere pattern is shown as a solid line.

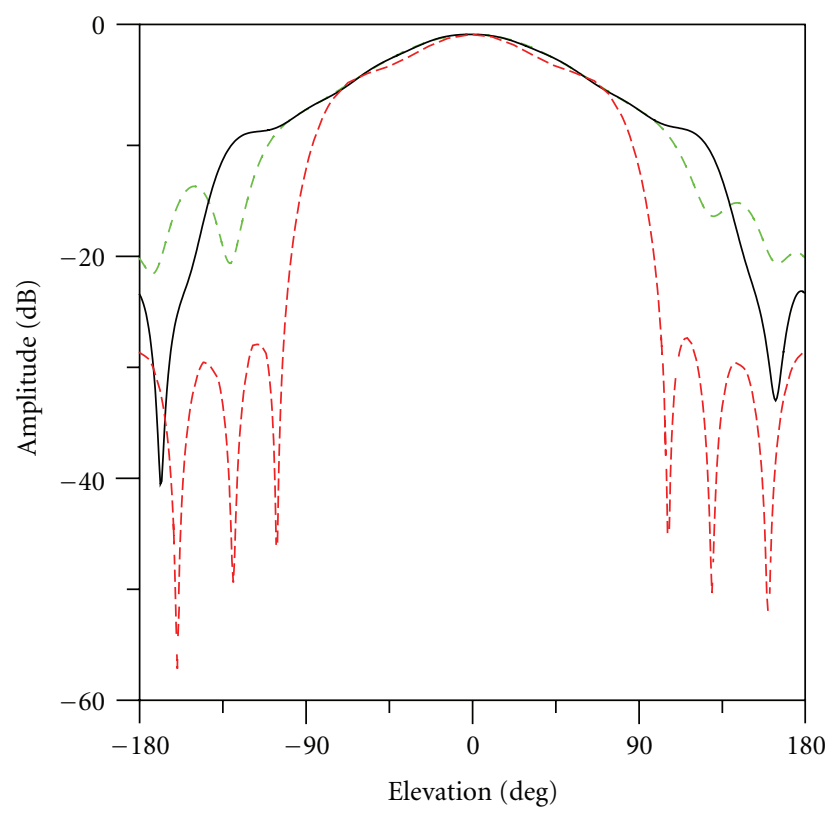

FIGURE 6: Rectangular waveguide probe: comparison of the fullsphere E-plane far-field patterns obtained using the zero-fill (long dash) and the least-squares (short dash) techniques. The full-sphere pattern is shown as a solid line.

approximate directivity, experience indicates that the error in $E$ usually will be less than $10 \%$.

\section{Summary and Future Work}

We have demonstrated a constrained least-squares technique for calculating the spherical mode coefficients of a small antenna from forward-hemisphere far-field data. This technique can significantly reduce truncation errors that arise 
when the standard algorithm is used with zero-fill in the backward-hemisphere. While the least-squares algorithm is not especially efficient, computational times are still acceptable for small antennas.

Our technique can also be used to process near-field data when reliable full-sphere measurements are not available. The method may be adapted to serve when measurements are made in the range $0 \leq \theta \leq \theta_{0}$, where $\theta_{0}$ is not restricted to $90^{\circ}$. We are considering an iterative algorithm to find both $\mathbf{x}$ and $\lambda$ simultaneously. For well-conditioned systems, this could effectively reduce the computational complexity to $O\left(N^{3}\right)$. By beginning with a spherical-wave expansion and projecting onto a scanning surface, our method should be useful for mitigation of truncation error in planar and cylindrical scanning measurements, as well.

\section{Acknowledgments}

The authors thank Allen Newell, consultant, for bringing this problem to our attention. They also thank Brad Alpert of NIST for insightful discussions concerning the numerical methods employed in this work.

\section{References}

[1] M. H. Francis and R. C. Wittmann, "Near-field scanning measurements: theory and practice," in Modern Antenna Handbook, C. A. Balanis, Ed., chapter 19, John Wiley \& Sons, New York, NY, USA, 2008.

[2] E. B. Joy, C. A. Rose et al., "Windows 96 for planar nearfield measurements," in Proceedings of the 18th AMTA Annual Meeting \& Symposium, pp. 80-85, October 1996.

[3] P. R. Rousseau, "The development of a near-field data window function for measuring standard gain horns," in Proceedings of the 19th AMTA Annual Meeting \& Symposium, pp. 96-101, November 1997.

[4] O. M. Bucci and M. D. Migliore, "A new method for avoiding the truncation error in near-field antennas measurements," IEEE Transactions on Antennas and Propagation, vol. 54, no. 10, pp. 2940-2952, 2006.

[5] K. T. Kim, "Slepian transverse vector spherical harmonics and their application to near-field scanning," in Proceedings of the IEEE Antennas and Propagation Society, AP-S International Symposium, pp. 2138-2141, Spokane, Wash, USA, July 2011.

[6] J. D. Jackson, Classical Electrodynamics, John Wiley \& Sons, New York, NY, USA, 2nd edition, 1975.

[7] R. C. Wittmann, B. K. Alpert, and M. H. Francis, "Near-field, spherical-scanning antenna measurements with nonideal probe locations," IEEE Transactions on Antennas and Propagation, vol. 52, no. 8, pp. 2184-2186, 2004.

[8] R. C. Wittmann and C. F. Stubenrauch, "Spherical near-field scanning: Experimental and theoretical studies," NISTIR 3955, National Institute of Standards and Technology, 1990.

[9] R. C. Wittmann, C. F. Stubenrauch, and M. H. Francis, "Spherical scanning measurements using truncated data sets," in Proceedings of the 24th AMTA Annual Meeting \& Symposium, pp. 279-283, November 2002.

[10] G. H. Golub and C. F. Van Loan, Matrix Computations, The Johns Hopkins University Press, Baltimore, Md, USA, 1989. 

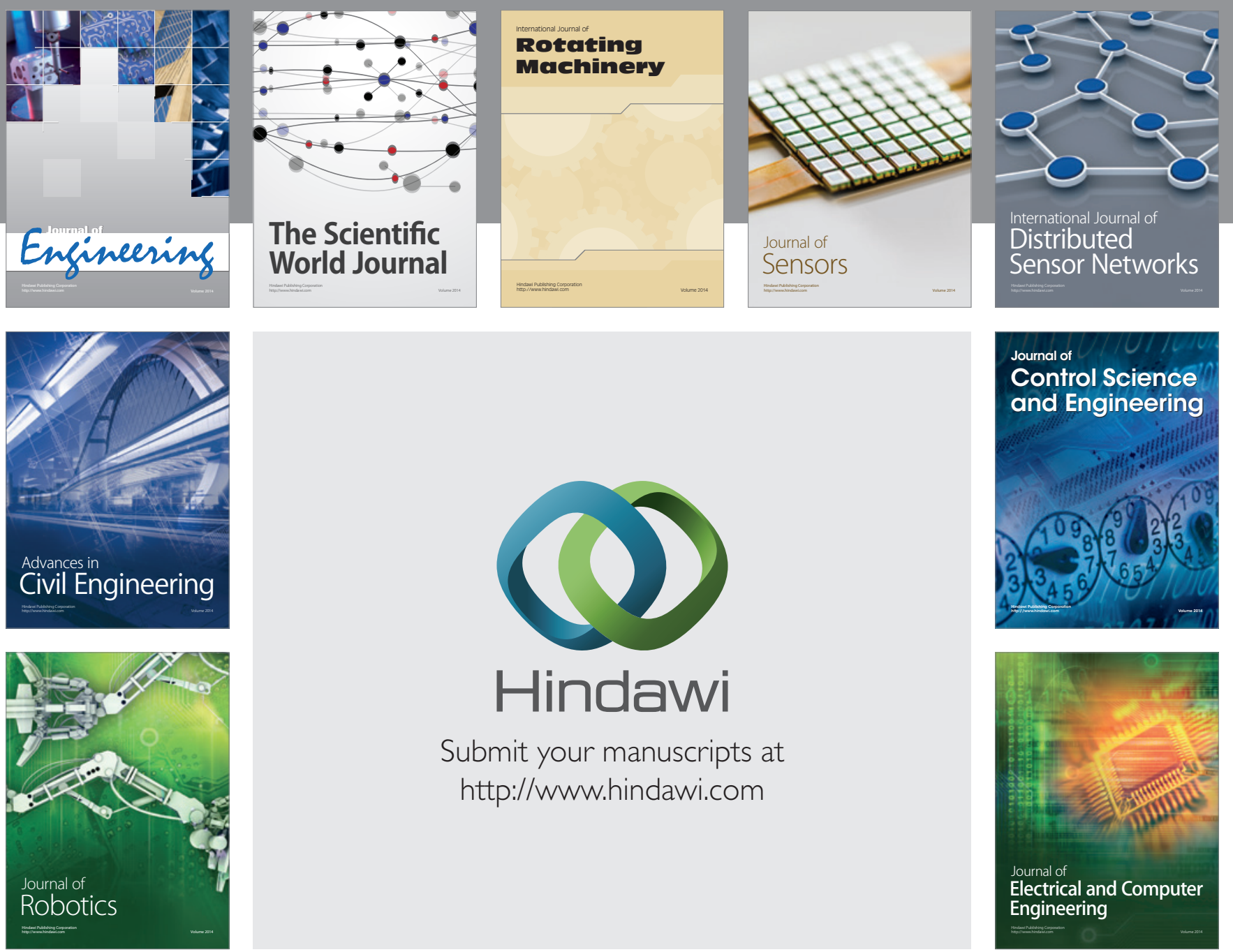

Submit your manuscripts at

http://www.hindawi.com
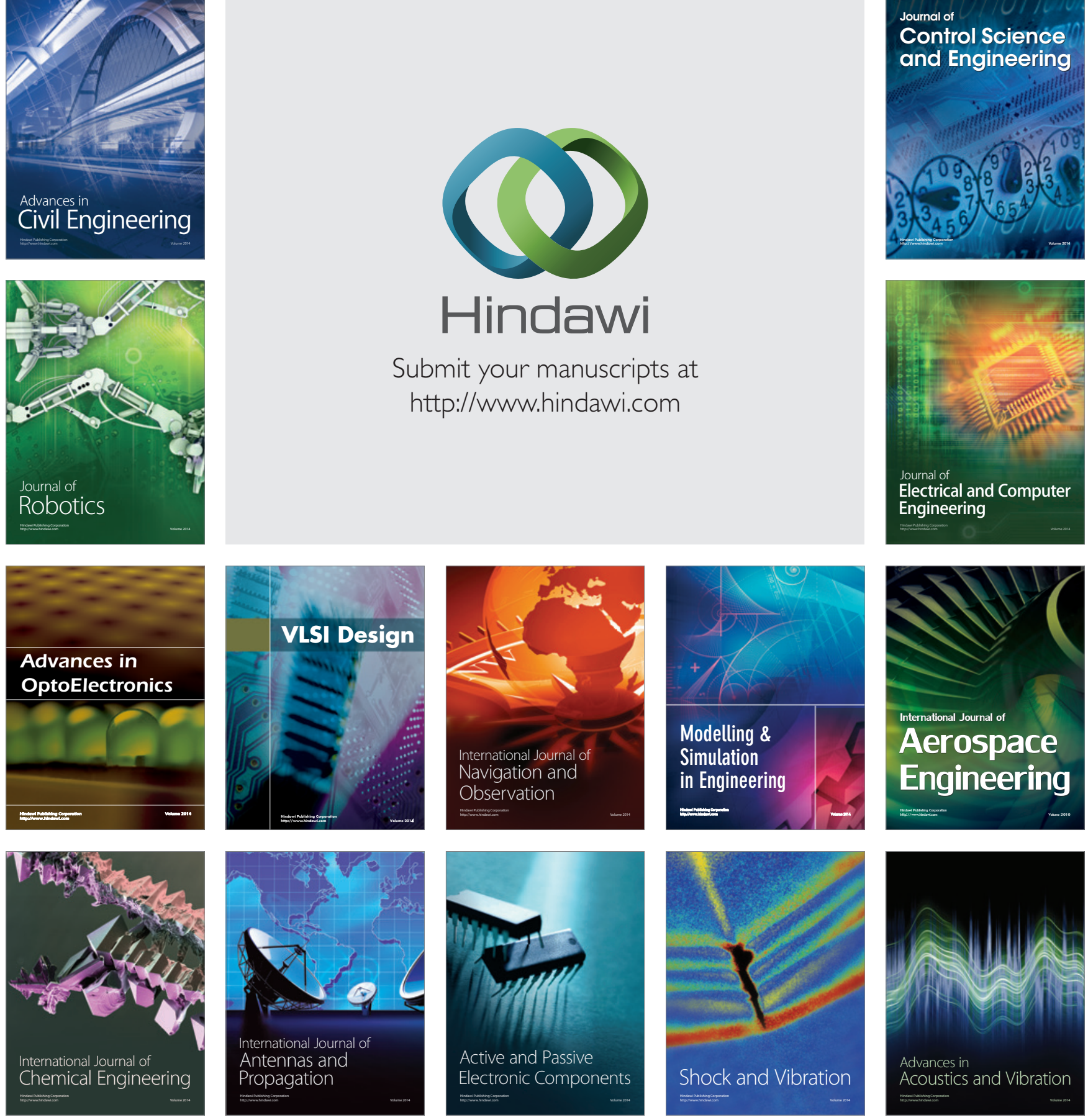